2.4 to $5.311-13$. Higher baseline scores require larger raw changes to represent clinically important differences 14 . Primary aim: To determine efficacy of intranasal ketamine in reducing cancer related pain. A clinical trial will be conducted to determine effect of intranasal ketamine on cancer related pain. Pain scores will be recorded on Numerical Pain Rating Scale (NPRS) at regular intervals throughout the study. Minimal clinically important differences (MCIDs) for pain ratings varies substantially based on patient population and statistical technique used, range of 0.4 to 3.7 points has been reported as a MCID. In general, improvements of pain severity $</=1.5$ points on NPRS could be seen as clinically irrelevant 9-13. Above that value, the cutoff point for "clinical relevance" depends on patients' baseline pain severity, and ranges from 2.4 to 5.3 11-13. Higher baseline scores require larger raw changes to represent clinically important differences 14 . Several clinical trials for pain have reported a reduction of 2 points on NPRS to be clinically important.15-17 Therefore for the purposes of this study, MCID of 2 was used for sample size calculations. A prior research study done by Carr et al. studied effects of intranasal ketamine for breakthrough pain in patients with chronic pain of various etiologies. 18 Total number of subjects in this study was 20 (4 of these had cancer related pain).This study demonstrated a mean reduction of 2.7 units on NPRS $(\mathrm{P}<0.0001)$, with standard deviation of 1.87 . Since MCID is 2, effect size using this $(\mathrm{MCID} / \mathrm{SD})=1.05$. Power and sample size table: Assumptions: 1. T-test is the appropriate test (may not be the appropriate test since we have a small sample size and may not be able to assume normality of means based on the central limit theorem) 2. Distribution of reductions in pain score is normal 3. Effect size of 1.05 is clinically meaningful; Sample Size: A sample size of 7 from a population of 20 (in the study done by Carr et al.) achieves $80 \%$ power to detect a NPRS difference of -2 between the null hypothesis mean of 0.0 and the alternative hypothesis mean of 2 with an estimated standard deviation (SD) of 1.87 and with a significance level (alpha) of 0.05 using paired t-test assuming that the actual distribution is normal. We will include 10 patients to account for the possibility that the observed pain reduction in the current study may be different than the study done by Carr, as in this study patients were given ketamine for breakthrough pain, as opposed to for baseline pain. We will enroll 25 patients in the study to account for potential dropouts. RESULTS/ANTICIPATED RESULTS: Majority of subjects experienced the largest decrease in their pain with the 10mg IV dose. Side effects included nausea/vomiting and a feeling of unreality. All side effects resolved by the end of each study visit. No severe adverse events occurred. DISCUSSION/SIGNIFICANCE OF IMPACT: Further study is required to elucidate safety of NAS ketamine with long term use for cancer related pain.

3178

Effects of Motor Skill Training vs. Strength and Flexibility Exercise on Functional Limitations, Pain, and Movement Characteristics in People with Chronic Low Back Pain

Quenten L Hooker ${ }^{1}$, Kristen Roles ${ }^{1}$, Vanessa M. Lanier ${ }^{1}$ and Linda R. Van Dillen ${ }^{1}$

${ }^{1}$ Washington University in St. Louis

OBJECTIVES/SPECIFIC AIMS: Compare the short- and long-term effects of 2 treatments, MST and SF, on limitations in function, pain, and movement characteristics. The movement characteristics included the amount of early excursion ( $1^{\text {st }}$ half of decent) of the knee, hip, and lumbar spine during a functional activity test of picking up an object. METHODS/STUDY POPULATION: 154 participants were randomized to 6, 1-hour treatment sessions (once/ week for 6 weeks) of MST or SF. The MST group received individualized training to modify pain-provoking altered movement patterns during functional activities. The SF group received exercises for trunk strength and trunk and limb flexibility. At baseline, post-treatment and 6-month follow-up participants completed the modified Oswestry Disability Questionnaire (MODQ, a functional limitation measure; 0-100\%), the Numeric Pain Rating Scale (NRS, average pain prior 7 days; $0-10$ ) and a standardized pick up an object test, where sagittal plane knee, hip and lumbar spine excursion were calculated using $3 \mathrm{D}$ motion capture. A mixed model repeated measures ANOVA was used to examine the following effects: Treatment group (Tx), Time and Tx X Time for each self-report and movement variable. When the ANOVA was significant $(\mathrm{p}<0.05)$, a priori planned contrasts were examined. RESULTS/ANTICIPATED RESULTS: There was a significant Tx X Time interaction $(\mathrm{p}<0.01)$ for each outcome. Baseline: MST and SF were similar in MODQ scores $[\Delta 0.4 \%(-3.4-2.9)]$, NRS $[\Delta 0.0(-0.6-0.6)]$, knee $\left[\Delta 2.2^{\circ}\right.$ $(-6.7-2.5)]$, hip $\left[\Delta 0.4^{\circ}(-2.9-2.5)\right]$, and lumbar spine $\left[\Delta 0.1^{\circ}\right.$ $(-1.4-1.2)]$ early excursion. Post-Treatment: Both group's MODQ and NRS scores decreased ( $\mathrm{p}<0.01)$, but MST had a greater reduction in MODQ scores $[\Delta-7.6 \%(-3.9--11.0)]$ and lower average NRS scores $[\Delta-0.8(-0.1--1.4)]$ compared to SF. MST changed knee $\left[\Delta+18.6^{\circ}(14.6-22.1)\right]$, hip $\left[\Delta+10.8^{\circ}(8.5-13.1)\right]$, and lumbar spine $\left[\Delta-2.0^{\circ}(-3.0--1.0)\right]$ early excursion, while SF did not change early joint excursion (all $\mathrm{p}>0.72$ ). 6-Month Follow-up: The differences between MST and SF were maintained for all outcomes $(\mathrm{p}>0.26)$. DISCUSSION/SIGNIFICANCE OF IMPACT: MST was more effective at reducing functional limitations and pain and improving movement patterns during a functional activity compared to SF. For all variables, the differences identified during treatment between MST and SF were maintained at 6-month follow-up. Therefore compared to SF, MST that targets performance of altered movement patterns during functional activities appears to be superior for attaining and maintaining changes in functional limitations, pain, and movement characteristics in people with CLBP.

3010

\section{Effects of non-invasive brain stimulation on speech fluency and brain activity in adults who stutter: a randomized controlled clinical trial}

Emily O'Dell Garnett, Ph.D., CCC-SLP ${ }^{1}$, Soo-Eun Changv, Benjamin Hampstead ${ }^{1}$ and Ho Ming Chow ${ }^{1}$

${ }^{1}$ University of Michigan School of Medicine

OBJECTIVES/SPECIFIC AIMS: The goal of this study is to measure speech fluency and brain activity before and after 5 days of behavioral speech fluency training alone (sham group) or speech training plus stimulation (active group). A 1-month follow up will also be completed. The first primary outcome measure is changes in brain activation in speech motor control/timing network. The second primary outcome measure is changes in percentage of stuttered syllables during speech sample (speech fluency). The secondary outcome measure is changes from baseline on the Overall Assessment of Speakers Experience of Stuttering (OASES), a detailed subject rating of how stuttering affects their lives. METHODS/STUDY POPULATION: This study is a between subjects, counterbalanced, sham-controlled, double-blind design. Participants will be 40 adults who stutter who will be randomized (using minimization) into either the active or 
sham stimulation group, with all other study procedures being the same in both groups. Participants will completed 2 days of baseline testing, 5 consecutive days of brain stimulation during speech training, 2 days of post-testing, and a 1-month follow up. All outcome measures will be completed immediately before and after the 5 days of brain stimulation, as well as at follow-up. as of submission, 10 subjects have completed the study. Data collection is ongoing. RESULTS/ANTICIPATED RESULTS: Expected results. Questions this study aims to answer: 1) Does a more intensive training period lead to decreased stuttering? We expect that both groups will show improvements in speech fluency immediately after training. We expect that those in the active group will continue to exhibit improved speech fluency at 1 month follow up. 2) Does a more intensive training period lead to changes in brain activity? We expect that both groups will exhibit increased activity in auditory/motor regions immediately after training. We expect that the active group will continue to exhibit an increase in activity in these regions at 1 month follow up. DISCUSSION/SIGNIFICANCE OF IMPACT: This is the first RCT study involving brain stimulation in adults who stutter. We expect to provide preliminary evidence for the effectiveness of tDCS as an augmentative agent for increased speech fluency in adults who stutter during a brief, intensive training paradigm. We also expect to be able to provide information on the effects of tDCS on brain activity in speech and auditory-motor regions of the brain. The findings will add to the growing body of literature suggesting that developmental stuttering is a neurodevelopmental disorder with roots in timing and rhythmic aspects of speech motor control and auditory-motor integration.

\section{6}

\section{Estimation of the Prevalence of Cesarean Delivery for the} Extremely Preterm Fetus in Breech Presentation

Alissa Dangel ${ }^{1}$, Janis L Breeze ${ }^{1}$, Gordon Huggins ${ }^{1}$, Michael House ${ }^{2}$ and Kumaran Kolandaivelu ${ }^{3}$

${ }^{1}$ Tufts University; ${ }^{2}$ Tufts Medical Center and ${ }^{3} \mathrm{MIT}$

OBJECTIVES/SPECIFIC AIMS: Cesarean delivery is typically performed in the extremely preterm period ( 23 to 28 weeks) when the fetus is in breech presentation to avoid the potential risk of head entrapment by an insufficiently dilated cervix during a vaginal delivery. Assessment of the prevalence of extremely preterm breech cesarean delivery would help to appropriately guide future clinical interventions designed to increase the feasibility of vaginal delivery for this sub-group of patients. METHODS/STUDY POPULATION: We performed a cross-sectional study of the 2106 U.S. National Vital Statistics birth certificate database to estimate the prevalence of cesarean deliveries performed during the period of gestation from 23 to 28 weeks with a fetus in breech presentation. RESULTS/ ANTICIPATED RESULTS: An analysis of the total births in the 2016 registry $(3,945,875)$ was performed. The gestational age was limited to the target range of $230 / 7$ to $276 / 7$ weeks. Multiple gestation deliveries were excluded. This yielded 16,092 births of which 4,849 were noted to have breech presentation. The proportion of cesarean deliveries performed for singleton breech fetuses at this gestational range was $87 \%(4,203 / 4,849)$. DISCUSSION/ SIGNIFICANCE OF IMPACT: The probability of undergoing a cesarean delivery for an extremely preterm fetus in breech presentation is notably higher $(87 \%)$ when compared to an overall cesarean delivery rate of $31.9 \%$. Specific interventions to allow for vaginal delivery in this particular sub-group of the obstetric population would be useful to reduce maternal morbidity by increasing vaginal deliveries. Future work will attempt to address innovative solutions to prevent head entrapment by the cervix in this particular population and ultimately avoid cesarean delivery.

3167

\section{Evaluation of risk factors for progression from carbapenem-resistant Enterobacteriaceae bacteriuria to an invasive infection}

Jessica Howard-Anderson ${ }^{1}$, Rebekah Blakney ${ }^{2}$, Christopher Bower ${ }^{1}$, Mary Elizabeth Sexton ${ }^{1}$, Sarah W. Satola ${ }^{1}$, Monica M. Farley ${ }^{1}$ and Jesse T. Jacob ${ }^{1}$

${ }^{1}$ Emory University and ${ }^{2}$ Georgia Emerging Infections Program

OBJECTIVES/SPECIFIC AIMS: To describe the epidemiology of patients with carbapenem-resistant Enterobacteriaceae (CRE) bacteriuria in metropolitan Atlanta, GA and to identify risk factors associated with progression to an invasive CRE infection. We hypothesize that having an indwelling urinary catheter increases the risk of progression. METHODS/STUDY POPULATION: The Georgia Emerging Infections Program (EIP) performs active population- and laboratory-based surveillance to identify CRE isolated from a sterile site (e.g. blood) or urine among patients who reside in the 8-county metropolitan Atlanta area (population $\sim 4$ million). The Georgia EIP performs a chart review of each case to extract data on demographics, culture location, resistance patterns, healthcare exposures, and other underlying risk factors. We used a retrospective cohort study design to include all Georgia EIP cases with Escherichia coli, Klebsiella pneumoniae, Klebsiella oxytoca, Enterobacter cloacae, or Klebsiella (formerly Enterobacter) aerogenes, adapting the current EIP definition of resistance to only include isolates resistant to meropenem, imipenem or doripenem (minimum inhibitory concentration $\geq 4$ ) first identified in a urine culture from $8 / 1 / 2011$ to $7 / 31 / 2017$. Patients with CRE identified in a sterile site culture prior to a urine culture will be excluded. Within this cohort, we will identify which patients had a subsequent similar CRE isolate identified from a sterile site between one day and one year after the original urine culture was identified (termed "progression"). CRE isolates will be defined as similar if they are the same species and have the same carbapenem susceptibility pattern. Univariable analyses using T-tests or other nonparametric tests for continuous variables, and Chi-square tests (or Fisher's exact tests as appropriate) for categorical variables will compare patient demographics, comorbidities and presence of invasive devices including urinary catheters between patients who had progression to an invasive infection and those who did not have progression. Covariates with a p-value of $<0.2$ will be eligible for inclusion in the multivariable logistic regression model with progression to invasive infection as the primary outcome. All statistical analyses will be done in SAS 9.4. RESULTS/ ANTICIPATED RESULTS: From 8/1/2011 to 7/31/2017 we have preliminarily identified 546 patients with CRE first identified in urine, representing an annual incidence rate of 1.1 cases per 100,000 population. Most cases were K. pneumoniae (352, 64\%), followed by E. coli $(117,21 \%)$, E. cloacae $(48,9 \%)$, K. aerogenes $(18,3 \%)$, and $\mathrm{K}$. oxytoca $(11,2 \%)$. The mean patient age was $64+/-18$ years and the majority $(308,56 \%)$ were female. Clinical characterization through chart review was available for 507 patients. The majority of the patients were black $(301,59 \%)$, followed by white $(166,33 \%)$, Asian (12, 2\%), and other or unknown race (28, 6\%). 466 (92\%) patients had at least one underlying comorbid condition with a median Charlson Comorbidity Index of 3 (IQR 1-5). 460 (91\%) infections were considered healthcare-associated (366 community-onset 